\title{
Unique fine scale village spatial-temporal distributions of Anopheles farauti differ by physiological state and sex
}

\author{
Edgar J. M. Pollard ${ }^{1 *}$, Tanya L. Russell', Allan Apairamo ${ }^{2}$ and Thomas R. Burkot ${ }^{1}$
}

\begin{abstract}
Background: The ecology of many mosquitoes, including Anopheles farauti, the dominant malaria vector in the southwest Pacific including the Solomon Islands, remains inadequately understood. Studies to map fine scale vector distributions are biased when trapping techniques use lures that will influence the natural movements of mosquitoes by attracting them to traps. However, passive collection methods allow the detailed natural distributions of vector populations by sex and physiological states to be revealed.
\end{abstract}

Methods: The barrier screen, a passive mosquito collection method along with human landing catches were used to record An. farauti distributions over time and space in two Solomon Island villages from May 2016 to July 2017.

Results: Temporal and spatial distributions of over 15,000 mosquitoes, including males as well as unfed, host seeking, blood-fed, non-blood fed and gravid females were mapped. These spatial and temporal patterns varied by species, sex and physiological state. Sugar-fed An. farauti were mostly collected between 10-20 m away from houses with peak activity from 18:00 to 19:00 h. Male An. farauti were mostly collected greater than $20 \mathrm{~m}$ from houses with peak activity from 19:00 to 20:00 h.

Conclusions: Anopheles farauti subpopulations, as defined by physiological state and sex, are heterogeneously distributed in Solomon Island villages. Understanding the basis for these observed heterogeneities will lead to more accurate surveillance of mosquitoes and will enable spatial targeting of interventions for greater efficiency and effectiveness of vector control.

Keywords: Anopheles farauti, Barrier Screen, Solomon Islands, Males, Sugar-feds

\section{Background}

Mosquito ecology remains inadequately understood for many species [1, 2], including Anopheles farauti, a dominant malaria vector in the southwest Pacific from western Indonesia through Papua New Guinea and the Solomon Islands to Vanuatu [3, 4]. Although there are behavioural differences among species [5], in general, mosquitoes fly to satisfy five basic behaviours: to blood feed, to find favourable resting sites, to lay eggs, to mate and to sugar feed [2]. Much is known about the blood-feeding of $A n$. farauti $[6,7]$ but less is known about resting $[6,8-10]$

\footnotetext{
*Correspondence: edgarjmp@gmail.com

${ }^{1}$ Australian Institute of Tropical Health and Medicine, James Cook University, Cairns, QLD 4870, Australia

Full list of author information is available at the end of the article
}

and oviposition behaviours [11-13]. These behaviours directly impact the efficacy of the three WHO recommended interventions of insecticide-treated nets (ITNs), indoor residual spray (IRS) and larval source management (LSM) [14]. Further, very little is known about two behaviours of An.farauti, sugar-feeding and mating, both of which are targets of novel vector control tools. There are no published data on where or on what plants An. farauti prefer to take sugar meals and An. farauti swarms have also not yet been observed.

There are significant variations in activity patterns among species and these patterns are changing as mosquitoes respond differently to selection pressures induced by vector control and changing environmental conditions $[15,16]$. Prior to IRS with DDT, An. farauti sought blood 
meals throughout the night, both indoors and outdoors. After the malaria elimination campaigns using IRS with DDT, a shift to earlier and more outdoor blood-feeding occurred. This behavioural shift was reinforced by the widespread deployment of ITNs to the point where $76 \%$ of biting now occurs outdoors before 21:00 $\mathrm{h}$ [3].

Knowledge of mosquito behaviours has been dominated by the use of traps with lures (including the use of humans and animals as baits) to define densities and distributions of species. Data generated in most traps provide useful "snapshots" on numbers of mosquitoes in specific physiological states, but these numbers may be biased by the lures used with traps. Thus, such data provides only limited insights into mosquitoes transitioning from one state to the next or where these behaviours take place (as lures induce mosquitoes to move towards the traps) [17]. There is a need to track mosquito behaviours without influencing the behaviours themselves to understand how best to monitor and control vector populations.

The barrier screen is an insecticide-free neutral (no bait or lure) net "trap" that intercepts mosquitoes as they fly in pursuit of blood meals, resting and oviposition sites, mating sites or sugar sources $[18,19]$. Mosquitoes when flying between blood-feeding, oviposition, mating, sugarfeeding and resting sites temporarily stop to rest when encountering a barrier screen, from which they can be collected. This approach is advantageous to studies of mosquito distributions in that it does not alter the natural locations of mosquitoes with lures and it samples both male and female mosquitoes of all physiological states. The natural outdoor temporal and spatial distributions of An. farauti subpopulations by sex and physiological status were mapped within villages in the Solomon Islands using barrier screens.

\section{Methods \\ Study sites}

The study was conducted in Jack Harbour village on Kolombangara Island in Western Province $\left(8.059^{\circ} \mathrm{S}\right.$, $157.196^{\circ} \mathrm{E}$ ) and Haleta village on Ngella Sule Island in Central Province $\left(9.098^{\circ} \mathrm{S}, 160.115^{\circ} \mathrm{E}\right)$ in the Solomon Islands (Fig. 1) [20]. Both coastal villages are on mountainous, rain-forested islands; with a mean daily temperature of $27^{\circ} \mathrm{C}$ and annual rainfall between $3000-5000 \mathrm{~mm}$ [21]. Haleta had a population of 366 in 70 households and Jack Harbour had a population of 151 in 38 households. Central Province had an annual parasite incidence (API) of 280 malaria cases per 1000 persons while Western Province had an API rate of 30 malaria cases per 1000 persons in 2016 [22].

Anopheles farauti, the dominant malaria vector in the Solomon Islands, is the only human-biting anopheline

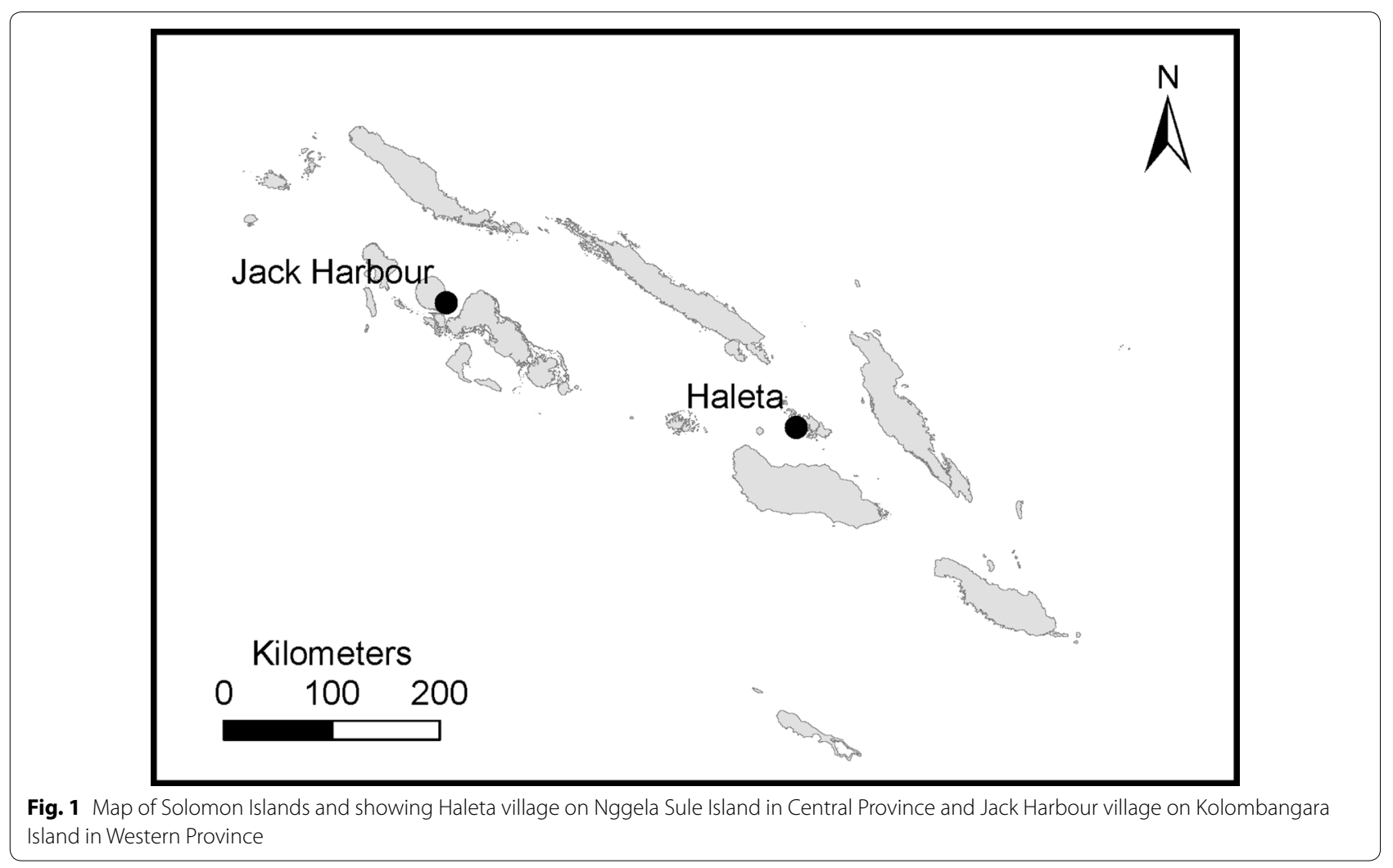


found in both villages with a mean of 14.8 bites per person per half-night $(\mathrm{b} / \mathrm{p} / \mathrm{h}-\mathrm{n})$ in Haleta village for $2011-2014$ [3] and $26.3 \mathrm{~b} / \mathrm{p} / \mathrm{h}-\mathrm{n}$ in Jack Harbour for 2014-2016 [20].

\section{Sampling adult mosquitoes}

During each sampling period, mosquitoes were sampled simultaneously over 4-6 sequential nights from 18:00 to 00:00 h using both barrier screens (BS) and human landing catch (HLC) in May, August and November 2016 and February 2017. In addition, Haleta was sampled in July 2017.

Barrier screens were constructed from $20 \mathrm{~m}$ long black high-density polyethylene shade cloth of $70 \%$ shading $\left(160 \mathrm{~g} / \mathrm{m}^{2}\right.$; Coolaroo ${ }^{\circledR}$ Gale Pacific Ltd, Melbourne, Australia) $[18,19]$. Mosquitoes resting on barrier screens were collected by mouth aspiration for 15 min every hour by collectors to whom insect repellent had been applied. For each mosquito, the time of collection by hour; the side of the barrier screen and resting height above the ground [using 3 broad categories of low $(0-0.6 \mathrm{~m})$, medium $(0.6-1.2 \mathrm{~m})$ and high $(1.2-1 . \mathrm{m})]$ were recorded. On any given night, 8 barrier screens were deployed across a village, and the distance of barrier screens to the nearest house and primary larval habitat measured. Barrier screens were relocated to sample a wide range of habitats/locations. Host-seeking females were also captured by HLC outdoors at 10 sites distributed throughout each village. The same locations were used for all HLC sampling efforts during all sampling periods as described previously $[3,20]$.

Captured anophelines were held by hour and collection station until identified to species by morphology [8], and categorised to sex (male or female) and physiological state at the field sites. Unfed, blood-fed and gravid mosquitoes were identified according to Detinova [23]; mosquitoes with a distended abdomen with a clear, likely sugar meal will hereafter be referred to as sugar-fed.

\section{Weather measurements}

Weather Meters (Kestrel 4500) with wind vanes recorded the temperature, humidity and wind speed and direction at ground level and at $1.8 \mathrm{~m}$ above the ground nightly during collections.

\section{Statistical analysis}

Generalised linear models (GLMs) with Gaussian distribution were used to analyse differences in (i) the temporal density of mosquitoes compared between physiological states; (ii) the distance from nearest house and physiological state; and (iii) the resting density of mosquitoes with average temperature, humidity and wind speed during collections. The significance of the interaction was analysed using a Chi-square test (ANOVA) that compared the fit of two nested Poisson GLM models. The effect of barrier screen height on resting female mosquito densities was analysed with a Generalized Linear Mixed Model (GLMM) with a negative binomial distribution and a random factor for the date (glmer.nb; package $=l m e 4$ ). Samples without any resting mosquitoes were removed from the analysis. Incorporating date as the random factor into the GLMM model accounted for natural fluctuations in mosquito densities observed while increasing the power of the model. This analysis was conducted using R statistical software (ver.3.1.2).

\section{Geospatial analysis}

Vector foci (areas with higher than mean densities) were determined using FleXScan (v3.1.2), a spatial Poisson distribution model to identify aggregated clusters by identifying spatial windows with greater ratios of observed to expected cases (relative risk). A single cluster detection was based on a spatial matrix defined using triangular irregular networks created based on Delaunay Triangulation, with Euclidian distance, limited to 10 stations with $P<0.01$. The FleXScan identified foci were then mapped in ArcMap 10.1 with a $10 \mathrm{~m}$ buffer.

\section{Results}

A total of 3411 mosquitoes resting on barrier screens were collected: 2345 from Jack Harbour during 21 half nights and 1066 from Haleta during 28 half nights of collections. The positions of barrier screen positions in Haleta and Jack Harbour are shown in Fig. 2.

Of these, 2292 were An. farauti and 1283 were culicines. Ninety-two percent of the An. farauti collected were females $(n=2121)$ and $87 \%$ of the culicines were females $(n=1119)$. Culicines were composed of a mix of species in the genera Culex $(C x$. sitiens, $C x$. quinquefasciatus) and Verallina spp. There were also occasional rare collections of Aedes (Ae. scutellaris) and Armigeres spp. on the barrier screens. Of the female An. farauti, $67 \%$ were unfed $(n=1421), 23 \%$ blood-fed $(n=484), 8 \%$ sugar-fed $(n=173)$ and $2 \%$ gravid $(n=43)$. Mean number of resting female An. farauti per barrier screen per halfnight $(\mathrm{r} / \mathrm{bs} / \mathrm{h}-\mathrm{n})$ during sampling periods ranged from $0.9 \mathrm{r} / \mathrm{bs} / \mathrm{h}-\mathrm{n}$ in the dry season to $11.0 \mathrm{r} / \mathrm{bs} / \mathrm{h}-\mathrm{n}$ in the wet season.

A total of 12,733 female, blood-seeking An. farauti were collected by HLC: 7296 from Jack Harbour (14 half nights) and 5437 from Haleta (20 half nights) villages over 34 half nights. Mean number of host-seeking female An. farauti per sampling period ranged from $1 \mathrm{~b} / \mathrm{p} / \mathrm{h}-\mathrm{n}$ to $13 \mathrm{~b} / \mathrm{p} / \mathrm{h}-\mathrm{n})$.

\section{Mosquito distributions on barrier screens}

The hourly numbers of mosquitoes collected on barrier screens varied by physiological status $\left(\chi^{2}=-205.37\right.$, 

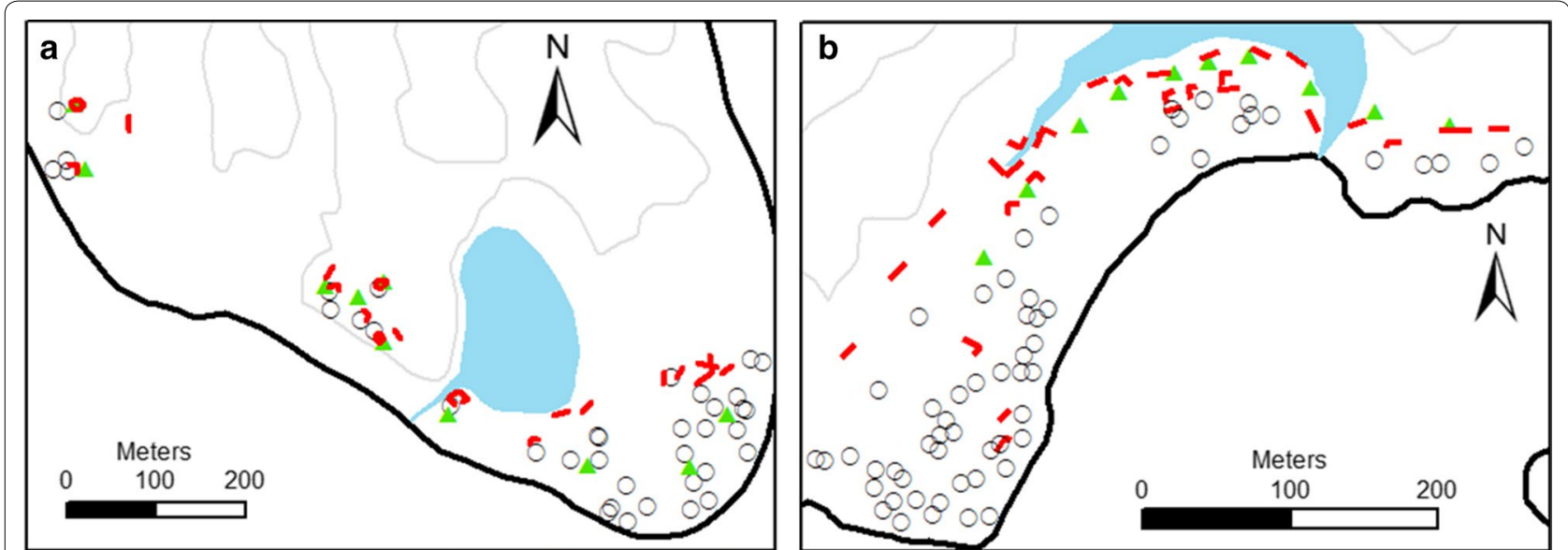

Fig. 2 Village maps of Jack Harbour (a) and Haleta (b) showing all locations where barrier screens (red) and human landing catch stations (green) were located, as well as houses (circles) and primary larval habitat (blue)
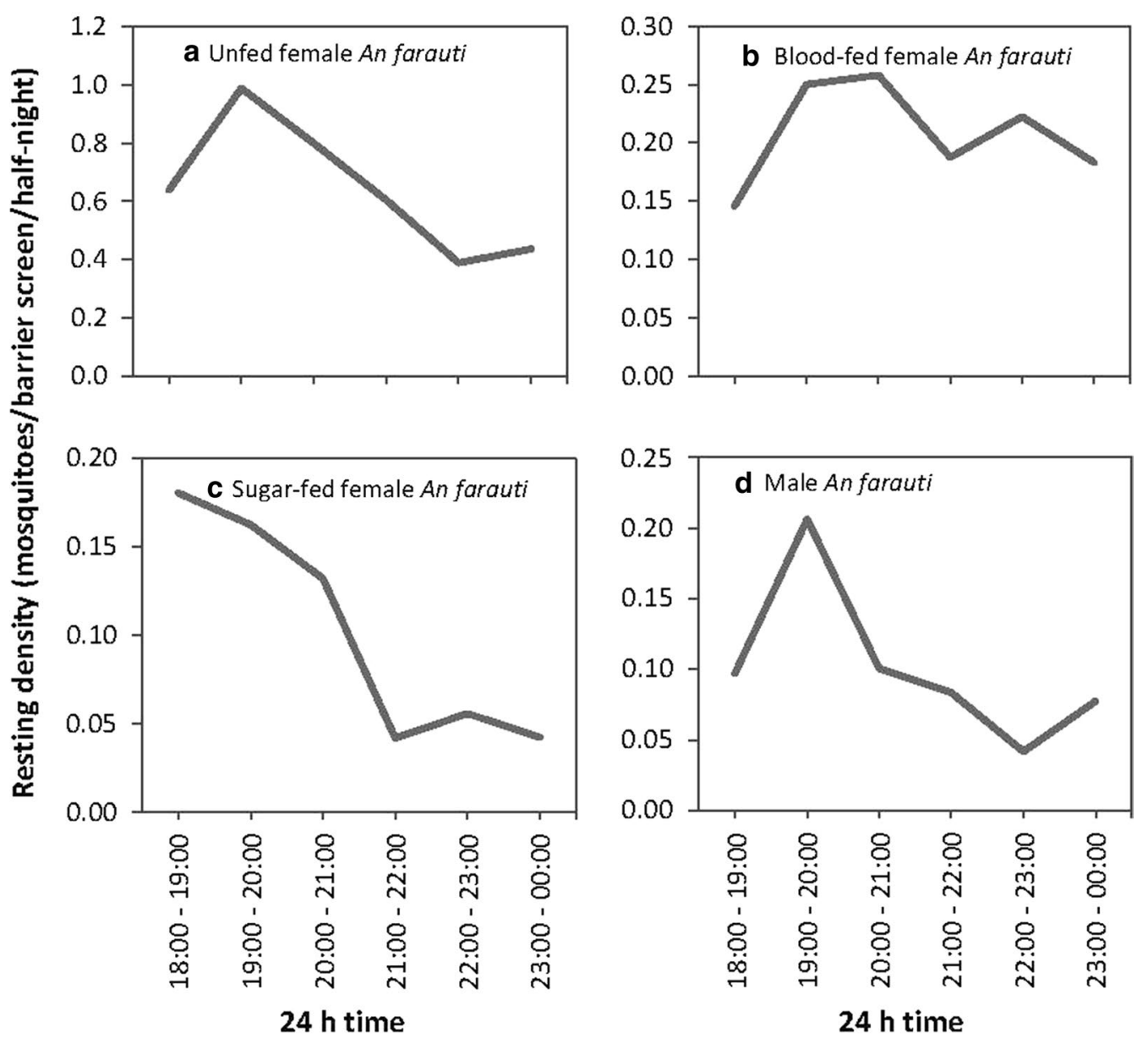

24 h time

Fig. 3 Densities of An. farauti on barrier screens by time. a Unfed females. $\mathbf{b}$ Blood-fed females. c Sugar-fed females. $\mathbf{d}$ Males

$d f=-36, P \leq 0.0001)$. Numbers of unfed female and male An. farauti resting on the barrier screens peaked at 19:00-20:00 $\mathrm{h}$, decreasing to 00:00 $\mathrm{h}$ when sampling ceased (Fig. 3). The number of blood-fed female $A n$. farauti on barrier screens maintained a longer peak (from 19:00-21:00 h) and had a more gradual decline 
in numbers to 00:00 h. Sugar-fed female An. farauti were collected earlier in the evening, peaking at 18:00-19:00 h. Blood-seeking An. farauti females from HLC had a similar temporal patterns to resting unfed females on the barrier screens as also recorded in previous studies in the same villages [3].

There was a significant inverse association between the height above the ground where mosquitoes were collected and the mean numbers collected ( $\beta=-0.3596, \mathrm{SE}=0.1331, P=0.007$ ): $57 \%$ of An. far auti females were collected within $60 \mathrm{~cm}$ of the ground with a mean of $3.8 \mathrm{r} / \mathrm{bs} / \mathrm{h}-\mathrm{n}$. In contrast, the mean resting density between 60 and $120 \mathrm{~cm}$ above the ground was $2.3 \mathrm{r} / \mathrm{bs} / \mathrm{h}-\mathrm{n}$. Above $120 \mathrm{~cm}$, only $1.2 \mathrm{r} / \mathrm{bs} / \mathrm{h}-\mathrm{n} A n$. farauti were collected.

\section{Geospatial resting locations Distance from house}

There was a significant interaction between the distance to the house and the physiological state of resting female An. farauti $\left(\chi^{2}=-136.82, d f=-4, P \leq 0.001\right)$. Unfed and blood-fed $A n$. farauti were most commonly collected within $10 \mathrm{~m}$ of a house, while more sugar-fed female An. farauti were collected 11-20 m from houses (Fig. 4). Male An. farauti numbers were highest at distances $>20 \mathrm{~m}$ from houses and also most (62\%) were
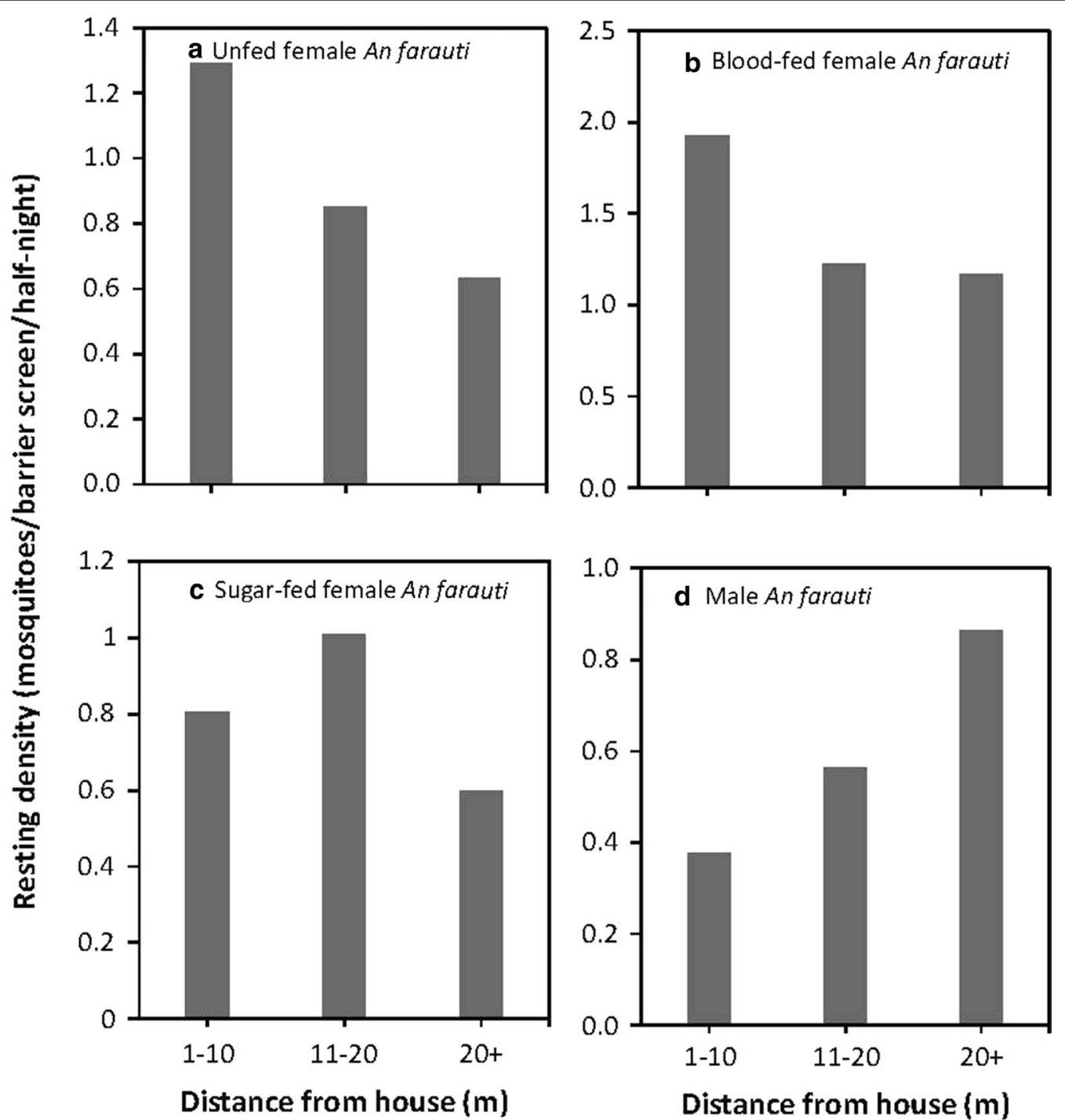

Fig. 4 Densities of An. farauti on barrier screens in proximity to the closest house. a Unfed females. b Blood-fed females. c Sugar-fed females. d Males 
collected within $10 \mathrm{~m}$ of a large swamp especially evident in Haleta village.

\section{Resting foci}

Significant foci of mosquitoes by physiological states and species were identified within each village (Table 1). In Haleta and Jack Harbour villages, there was high spatial overlap where unfed female An. farauti and sugar-fed and blood-fed female An. farauti were collected (Figs. 5, 6). Sugar-fed An. farauti females and An. farauti males were also collected in close proximity, particularly evident in Jack Harbour. Blood-seeking An. farauti foci also tended to resemble patterns of unfed and blood-fed female $A n$. farauti. Although there were differences between the villages, the male An. farauti foci was always smaller than the female foci. The populations of female culicines and anophelines (e.g. An. farauti) were largely segregated into different parts of the villages.

\section{Weather}

Temperature, humidity and wind speed strongly influenced mosquito numbers on barrier screens (temperature: $\beta=-0.4398, \quad \mathrm{SE}=0.0340, \quad P \leq 0.001$; humidity: $\beta=-0.1570, \quad \mathrm{SE}=0.0073, \quad P \leq 0.001$; wind speed: $\beta=-2.7890, \mathrm{SE}=0.1558, P \leq 0.001$ ) and with HLC (temperature: $\beta=-0.2922, \mathrm{SE}=1.8985, P \leq 0.001$; humidity: $\beta=0.0516, \quad S E=0.0108, P \leq 0.001$; wind speed: $\beta=-1.9914, \mathrm{SE}=0.2026, P \leq 0.001)$. Higher average wind speeds were associated with lower $A n$. farauti collections on barrier screens and with human landing catch (Fig. 7). Collection densities above 1 female $A n$. farauti per night on barrier screens never occurred when average wind speeds were $>0.2 \mathrm{~m} / \mathrm{s}$. Lower average humidity during mosquito sampling was associated with lower numbers of An. farauti collected on barrier screens. Higher densities of female An. farauti on barrier screens were associated with lower average temperatures.

\section{Discussion}

In villages, exposure to anophelines and malaria transmission is unevenly distributed [24]. Previous studies documented heterogeneity in the distribution of biting An. farauti in villages in the Solomon Islands and proposed that the risk of malaria was best estimated by biting rates in low-transmission villages [20]. This study expands our understanding of the distributions of mosquitoes to include other species, physiological states, sex and behaviours. Analogous to the temporal activity patterns of $A n$. farauti, the spatial activity patterns for $A n$. farauti in the Solomon Islands also differed by physiological states and sex. Whereas biting rates estimate the risk of malaria transmission, the distributions of other vectors by physiological states or behaviours may enable optimising control and monitoring strategies. This study also highlights the suitability of the barrier screen for collecting non-anopheline mosquitoes.

The complexity of the environment influences the locations where mosquitoes were sampled, with barrier screens near potential blood sources (houses) intercepting more unfed (potentially blood-meal seeking) and blood-fed An. farauti. The barrier screens were most efficient at sampling unfed mosquitoes (63-67\%), followed by blood-fed (23-36\%) with only $1-2 \%$ being gravid, as

Table 1 Spatial clusters (foci) of An. farauti and Culex within Jack Harbour and Haleta villages

\begin{tabular}{|c|c|c|c|c|c|c|}
\hline Species & Physiological state & $\begin{array}{l}\text { Maximum } \\
\text { distance (m) }\end{array}$ & $\begin{array}{l}\text { Percent of locations } \\
\text { (census areas) }\end{array}$ & $\begin{array}{l}\text { Observed percent of } \\
\text { mosquitoes }\end{array}$ & $\begin{array}{l}\text { Expected no. of } \\
\text { mosquitoes }\end{array}$ & $\begin{array}{l}\text { Relative } \\
\text { risk (obs/ } \\
\text { exp) }\end{array}$ \\
\hline \multicolumn{7}{|l|}{ Haleta village } \\
\hline An. farauti & Unfed female & 52 & $7(2 / 29)$ & $28(107 / 381)$ & 30 & 3.61 \\
\hline An. farauti & Blood-fed female & 141 & $14(4 / 29)$ & $33(54 / 165)$ & 31 & 1.75 \\
\hline An. farauti & Sugar-fed female & 185 & $17(5 / 29)$ & $47(28 / 60)$ & 12 & 2.26 \\
\hline An. farauti & Male & 0 & $3(1 / 29)$ & $39(53 / 136)$ & 11 & 4.74 \\
\hline Culex spp. & Female Culex & 128 & $21(6 / 29)$ & $74(211 / 287)$ & 48 & 4.44 \\
\hline An. farauti & Blood-seeking female (HLC) & 341 & $70(7 / 10)$ & $76(4123 / 5437)$ & 3806 & 1.08 \\
\hline \multicolumn{7}{|c|}{ Jack Harbour village } \\
\hline An. farauti & Unfed female & 368 & $18(4 / 22)$ & $59(583 / 987)$ & 223 & 2.61 \\
\hline An. farauti & Blood-fed female & 385 & $32(7 / 22)$ & $61(172 / 280)$ & 99 & 1.75 \\
\hline An. farauti & Sugar-fed female & 103 & $23(5 / 22)$ & $71(80 / 113)$ & 30 & 2.64 \\
\hline An. farauti & Male & 40 & $9(2 / 22)$ & $70(21 / 30)$ & 4 & 4.90 \\
\hline Culex spp. & Female Culex & 71 & $18(4 / 22)$ & $64(525 / 824)$ & 89 & 5.92 \\
\hline An. farauti & Blood-seeking female (HLC) & 407 & $30(3 / 10)$ & $58(4268 / 7296)$ & 2189 & 1.95 \\
\hline
\end{tabular}




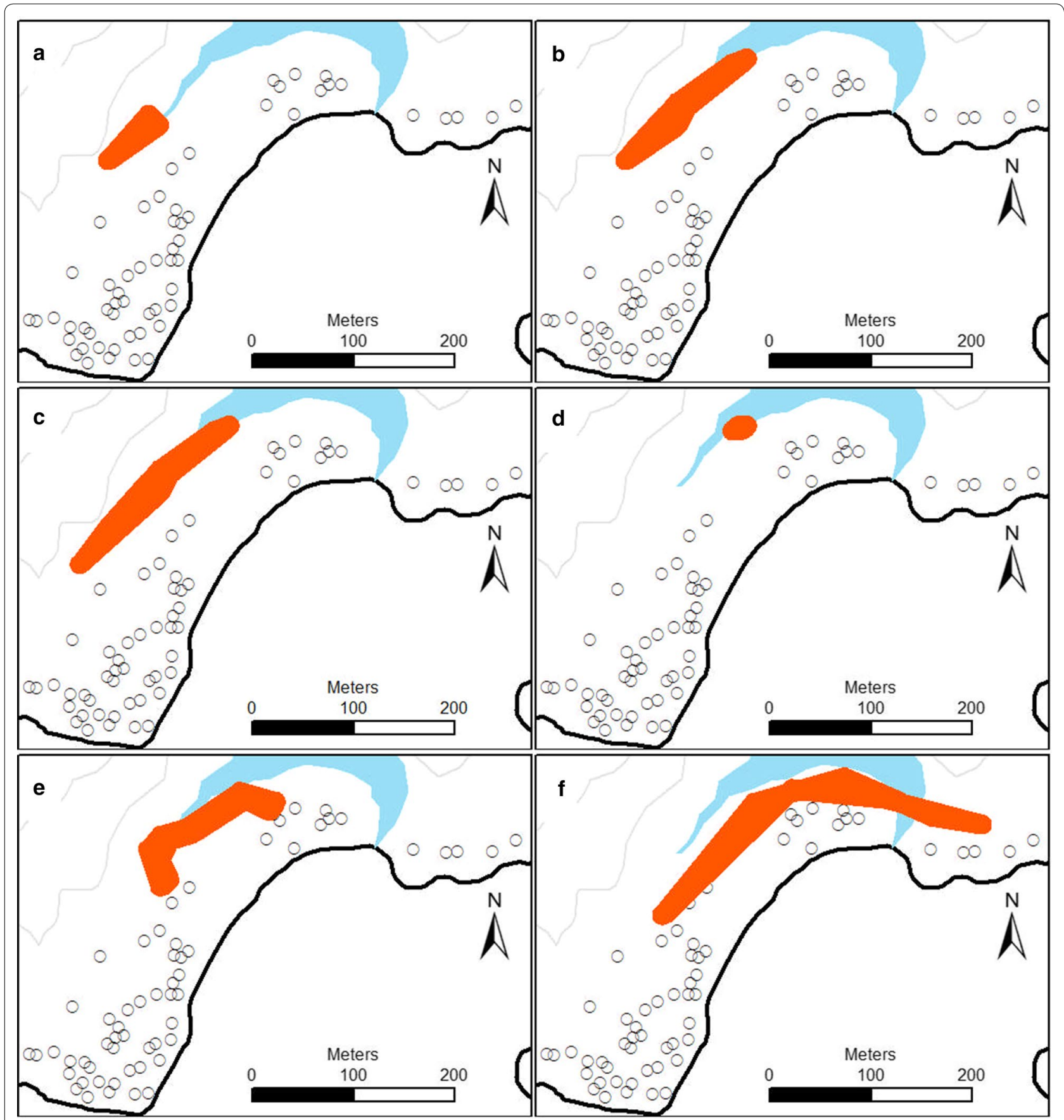

Fig. 5 Locations of significant mosquito foci (shown in orange) in Haleta village, Central Province, Solomon Islands, collected by barrier screens (a unfed female An. farauti; b blood-fed female An. farauti; c sugar-fed female An. farauti; d male An. farauti; e female culicine species) and human landing catch (f blood-seeking female An. farauti)

also seen from previous studies in PNG, Indonesia and the Solomon Islands $[25,26]$.

Here, the sugar-fed An. farauti were predominantly collected in the early evening, indicating that sugar-feeding is predominantly an early evening activity. This is similar to studies of the An. gambiae complex in Africa showing that sugar-feeding occurs early in the evening and morning (prior to blood-feeding) [27]. Generally, sugar-fed females and males, are collected but in low numbers $(<8 \%)$ on the barrier screens $[25,26]$, but with a high sampling effort sufficient numbers can be collected. 


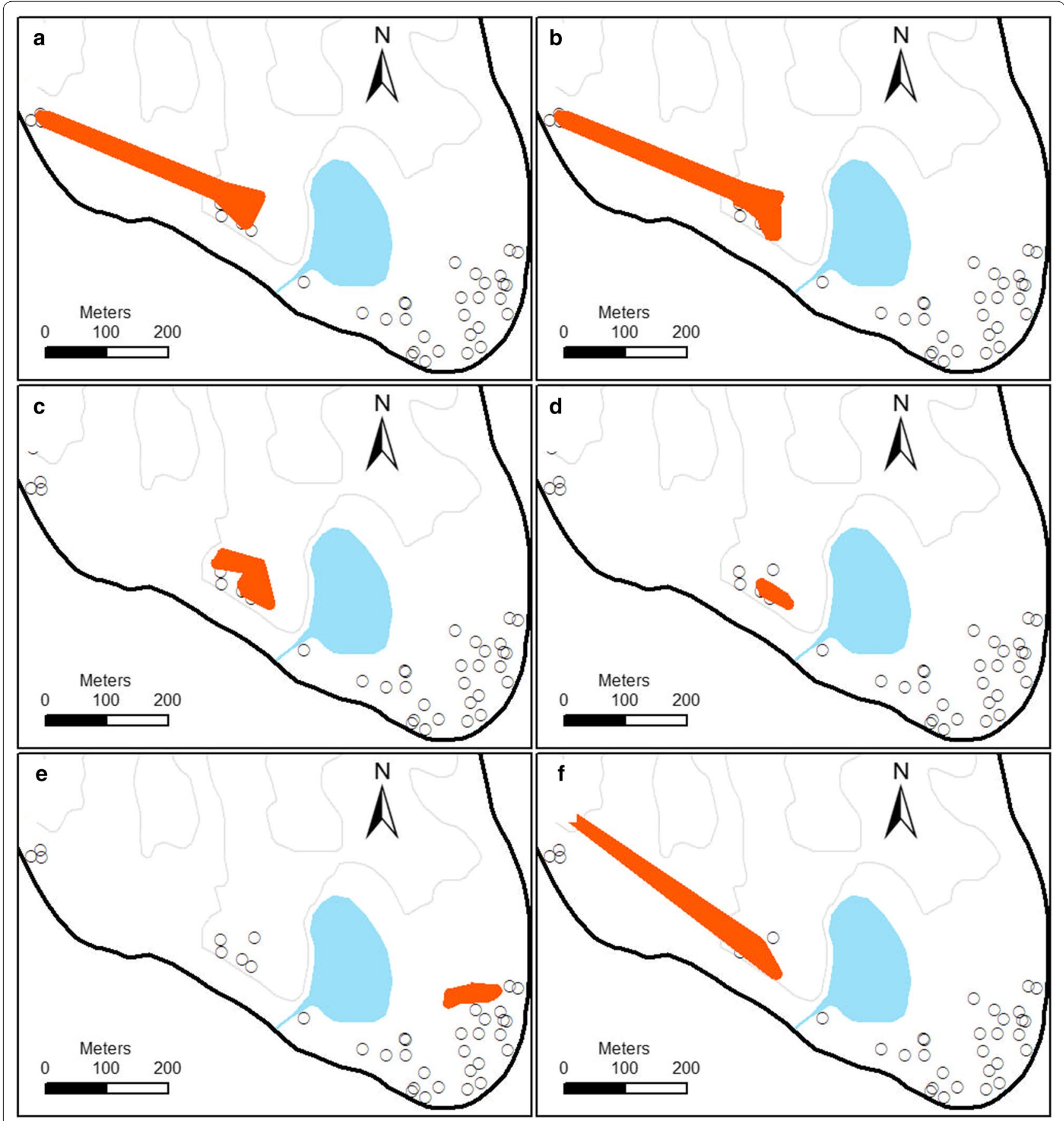

Fig. 6 Locations of significant mosquito foci (shown in orange) in Jack Harbour village, Western Province, Solomon Islands, collected by barrier screens (a unfed female An. farauti, b blood-fed female An. farauti, c sugar-fed female An. farauti, d male An. farauti, and e female culicine species) and human landing catch (f blood-seeking female An. farauti)

Across both villages, male An. farauti were collected in more geographically focused areas. Male $A n$. farauti were mostly collected on barrier screens near larval habitats shortly after sundown suggesting that emergence of males or swarming occurs in the early evening. This is the first indication of possible times and locations of An. farauti mating as swarms in this species have yet to be documented. 


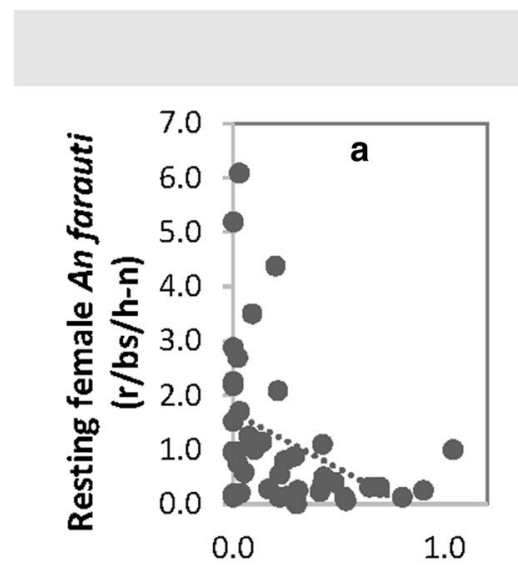

Wind Speed $(\mathrm{m} / \mathrm{s})$

\section{Barrier Screen}

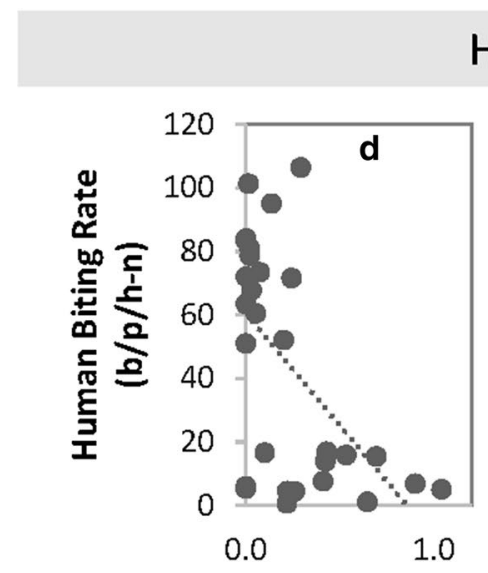

Wind Speed $\left(\mathrm{m} / \mathrm{s}^{\prime}\right.$
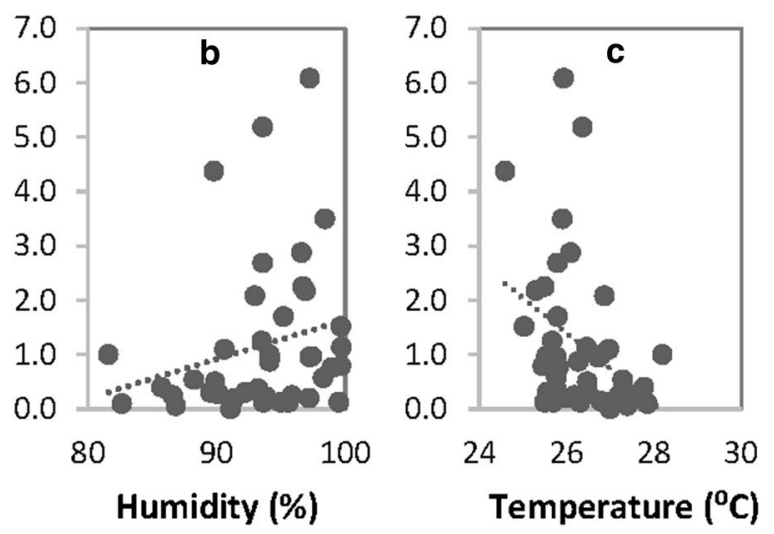

Human Landing Catch

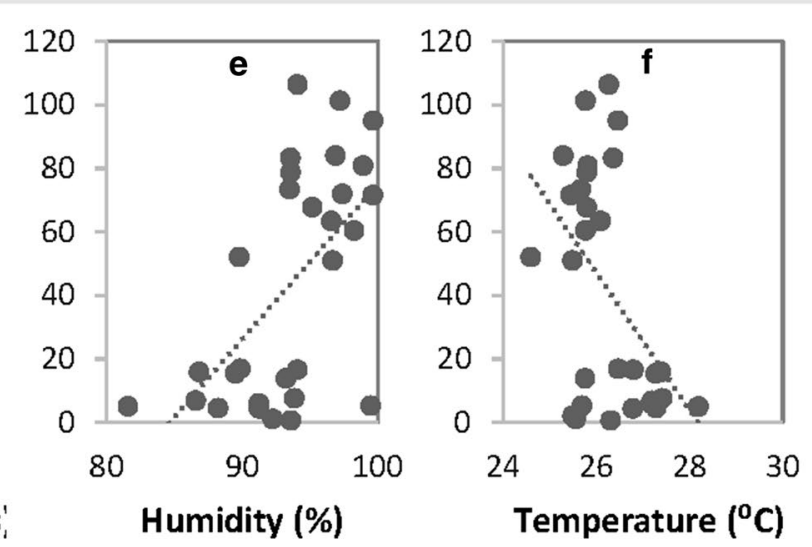

Fig. 7 Relationships between weather parameters and average total female resting An. farauti per half night with linear trendline. a Average wind speed and average total female resting An. farauti per half night. b Average humidity and average total female resting An. farauti per half night. c Average temperature and average total female resting An. farauti per half night. $\mathbf{d}$ Average wind speed and average total female biting An. farauti per half night. e Average humidity and average total female biting An. farauti per half night. f Average temperature and average total female biting An. farauti per half night

Mosquito population dynamics is strongly impacted by weather [27-29]. Wind, temperature and humidity are major factors influencing mosquito flight [30]. Despite the limited range in temperature, humidity and wind speed recorded during the present study, significant impacts on the densities of resting and biting $A n$. farauti were found: increased densities of biting and resting An. farauti were associated with higher humidities and lower temperatures (within the $24-30{ }^{\circ} \mathrm{C}$ range). The finding that wind speeds greater than 1 $\mathrm{km} / \mathrm{h}$ can significantly reduce $A n$. farauti flight is consistent with impact of wind on other species [31] and suggests that reductions in exposure to biting An. farauti can be obtained by avoiding protected areas in the early evening when most $A n$. farauti bites occur.
Data from outdoor barrier screens and indoor resting behaviours suggest many anophelines fly and rest predominantly within a meter of the ground $[19,25]$. The height above the ground where An. farauti were collected on barrier screens suggests that $A n$. farauti predominantly flies within a metre of the ground in the Solomon Islands. These observations are consistent with the observation that bites from An. farauti in Papua New Guinea were significantly reduced even at elevations of $35 \mathrm{~cm}$ [32].

These data defining the heights at which An. farauti fly and the influence of wind on flight suggest that significant protection from biting An. farauti can be afforded by two simple human behaviours: avoidance of protected areas in the early evening to maximise wind exposure (and thereby minimising mosquito bites) in the evening 
before sleeping and then sleeping in elevated houses under a LLIN. Spatial foci of An. farauti within the village were clearly different from culicine species and also differed in having different peak times of activities, signifying different ecological niches. The differing distributions of the culicines and anophelines suggests that interventions for controlling these different mosquitoes may require different distribution strategies.

This research provides fundamental bionomic information that can be utilised to support the optimisation of novel vector control tools. Attractive targeted sugar baits (ATSB) are an "attract and kill" strategy, where a highly attractive sugar lure is integrated into bait stations with an oral toxicant, usually garlic [33, 34]. In this study, foci of sugar-fed mosquitoes were mapped which could enable more effective placement of ATSBs. Insecticide treatment of barrier screens are an unproven technology that has the potential to kill mosquitoes who contact the screens, and warrants further research as a possible vector control tool.

\section{Conclusions}

Anopheles farauti subpopulations, as defined by physiological state and sex, were found to be heterogeneously distributed in Solomon Island villages. This heterogeneity is proposed to be a function of proximity to blood and sugar sources, as well as resting and oviposition sites with the density of mosquitoes at any given location moderated by weather parameters (temperature, humidity and wind). Understanding the basis for mosquito heterogeneities in villages will lead to more accurate surveillance of mosquitoes and greater efficiency and effectiveness of vector control tools. In the absence of new control tools, there are simple measures that individuals can take to protect themselves from mosquito bites based on an understanding of the factors that determine the distributions and densities of biting mosquitoes.

\section{Abbreviations \\ API: annual parasite incidence; b/p/h-n: bites/person/half-night; BS: barrier screen; HBR: human-biting rate; HLC: human landing catch; EIR: entomological inoculation rate; IRS: indoor residual spraying; LLIN: long-lasting insecticidal net; r/bs/n: resting/barrier screen/half-night.}

\section{Acknowledgements \\ Technical assistance when conducting the field work was provided by Jance Oscar and John Lodo formally of the Vector Borne Disease Control Pro- gramme, Solomon Islands. The authors thank the communities of Haleta and Jack Harbour villages as well as the support of Albino Bobogare, Director of National Vector Borne Disease Control Programme, Solomon Islands.}

\section{Authors' contributions}

EJMP and AA conducted the field collections. EJMP analysed the dataset and drafted the manuscript. TLR and TRB assisted with the experimental design, data analysis and writing the manuscript. All authors read and approved the final manuscript.

\section{Funding}

This work was supported by Grant No. 45114 from the Bill and Melinda Gates Foundation to the Malaria Transmission Consortium, and the National Institute of Allergy and Infectious Diseases of the National Institutes of Health for the International Centers of Excellence in Malaria Research in the Southwest Pacific (subaward U19AI08986 to James Cook University). EJMP was supported by a PhD scholarship from Rotarians Against Malaria.

\section{Availability of data and materials}

The datasets supporting the conclusions of this article are available in the JCU Tropical Data Hub repository: http://doi.org/10.25903/5d4a446668e70.

\section{Ethics approval and consent to participate}

Ethical approvals were obtained from the National Health Research \& Ethics Committee, Solomon Islands (2011-05-02, HRE002/16), the James Cook University Human Research Ethics Committee, Australia (H4914 and H6488). Meetings were held with community leaders, study participants and village residents, where the aims, the possible risks and potential benefits of the study were explained in Solomon Islands Pidgin. Mosquito collectors were then recruited from village residents and enrolled in the study after the risks were explained and obtaining informed written consent.

\section{Consent for publication}

Not applicable.

\section{Competing interests}

The authors declare that they have no competing interests.

\section{Author details}

${ }^{1}$ Australian Institute of Tropical Health and Medicine, James Cook University, Cairns, QLD 4870, Australia. ${ }^{2}$ National Vector Borne Disease Control Program, Honiara, Solomon Islands.

Received: 9 August 2019 Accepted: 19 November 2019

Published online: 26 November 2019

\section{References}

1. Ferguson HM, Dornhaus A, Beeche A, Borgemeister C, Gottlieb M, Mulla MS, et al. Ecology: a prerequisite for malaria elimination and eradication. PLoS Med. 2010;7:e1000303.

2. Service MW. Mosquito (Diptera: Culicidae) dispersal - the long and short of it. J Med Entomol. 1997;34:579-88.

3. Russell TL, Beebe NW, Bugoro H, Apairamo A, Chow WK, Cooper RD, et al. Frequent blood-feeding enables insecticide-treated nets to reduce transmission by mosquitoes that bite predominately outdoors. Malar J. 2016;15:156.

4. Beebe NW, Russell T, Burkot TR, Cooper RD. Anopheles punctulatus Group: evolution, distribution, and control. Annu Rev Entomol. 2015;60:335-50.

5. Verdonschot PFM, Besse-Lototskaya AA. Flight distance of mosquitoes (Culicidae): a metadata analysis to support the management of barrier zones around rewetted and newly constructed wetlands. Limnologica. 2014;45:69-79.

6. Burkot TR, Graves PM, Paru R, Lagog M. Mixed blood-feeding by the malaria vectors in the Anopheles punctulatus complex (Diptera: Culicidae). J Med Entomol. 1988;25:205-13.

7. Russell TL, Beebe NW, Bugoro H, Apairamo A, Cooper RD, Collins FH, et al. Determinants of host feeding success by Anopheles farauti. Malar J. 2016;15:152.

8. Belkin JN. The mosquitoes of the South Pacific (Diptera, Culicidae). Berkeley and Los Angeles: University of California Press; 1962.

9. Daggy $\mathrm{RH}$. The biology and seasonal cycle of Anopheles farauti on Espirtu Santo. New Hebrides. Ann Entomol Soc Am. 1945;38:3-13.

10. Chow CY. Ecology of malaria vectors in the Pacific. Cah ORSTOM ser Entomol Med Parasitol. 1969;7:93-7.

11. Russell TL, Burkot TR, Bugoro H, Apairamo A, Beebe NW, Chow WK, et al. Larval habitats of the Anopheles farauti and Anopheles lungae complexes in the Solomon Islands. Malar J. 2016;15:164. 
12. Sweeney AW. Larval salinity tolerances of the sibling species of Anopheles farauti. J Am Mosq Control Assoc. 1987;8:589-92.

13. Bugoro H, Hii J, Russell T, Cooper R, Chan B, Iro' ofa C, et al. Influence of environmental factors on the abundance of Anopheles farauti larvae in large brackish water streams in northern Guadalcanal. Solomon Islands. Malar J. 2011;10:262.

14. WHO. Guidelines for Malaria Vector Control. Geneva: World Health Organization; 2019.

15. Taylor B. Changes in the feeding behaviour of a malaria vector, Anopheles farauti Lav., following the use of DDT as a residual spray in houses in the British Solomon Islands Protectorate. Trans R Entomol Soc Lond. 1975;127:227-92.

16. Russell TL, Govella NJ, Azizi S, Drakeley CJ, Kachur SP, Killeen GF. Increased proportions of outdoor feeding among residual malaria vector populations following increased use of insecticide-treated nets in rural Tanzania. Malar J. 2011;10:80

17. Silver JB. Mosquito ecology: field sampling methods. 3rd ed. New York: Springer; 2008.

18. Burkot TR, Russell TL, Reimer $\sqcup$, Bugoro H, Beebe NW, Cooper RD, et al. Barrier screens: a method to sample blood-fed and host-seeking exophilic mosquitoes. Malar J. 2013;12:49.

19. Pollard EJM, Russell TL, Burkot TR. Maximising mosquito collections from barrier screens: the impacts of physical design and operation parameters Parasit Vectors. 2019:12:31.

20. Burkot TR, Bugoro H, Apairamo A, Cooper RD, Echeverry DF, Odabasi D, et al. Spatial-temporal heterogeneity in malaria receptivity is best estimated by vector biting rates in areas nearing elimination. Parasit Vectors. 2018;11:606.

21. Solomon Islands climate. https://www.met.gov.sb. Accessed 1 Jun 2019

22. Monitoring and Evaluation Unit. Solomon Islands Annual Malaria Programme Report 2016. In: Epidemiological \& Programmatic Report. Honiara, Solomon Islands: National Vector Borne Diseases Control Program, Ministry of Health \& Medical Services; 2017.

23. Detinova TS. Age-grouping methods in Diptera of medical importance with special reference to some vectors of malaria. Monogr Ser World Health Organ. 1962;47:13-191.

24. Lindsay SW, Schellenberg JRMA, Zeiler HA, Daly RJ, Salum FM, Wilkins HA. Exposure of Gambian children to Anopheles gambiae malaria vectors in an irrigated rice production area. Med Vet Entomol. 1995;9:50-8.
25. Davidson JR, Sukowati S, Shinta, Asih PSB, Syafruddin D, Baskin RN, et al. Using barrier screens to characterize mosquito composition, flight activity, and abdominal status in South Lampung, Indonesia. Parasit Vectors. 2018;11:440.

26. Keven JB, Katusele M, Vinit R, Koimbu G, Vincent N, Thomsen EK, et al. Species abundance, composition, and nocturnal activity of female Anopheles (Diptera: Culicidae) in malaria-endemic villages of Papua New Guinea: assessment with barrier screen sampling. Malar J. 2019;18:96.

27. Grover-Kopec EK, Blumenthal MB, Ceccato P, Dinku T, Omumbo JA, Connor SJ. Web-based climate information resources for malaria control in Africa. Malar J. 2006;5:38.

28. Wardrop NA, Barnett AG, Atkinson J-A, Clements AC. Plasmodium vivax malaria incidence over time and its association with temperature and rainfall in four counties of Yunnan Province, China. Malar J. 2013;12:452.

29. Gunda R, Chimbari MJ, Shamu S, Sartorius B, Mukaratirwa S. Malaria incidence trends and their association with climatic variables in rural Gwanda, Zimbabwe, 2005-2015. Malar J. 2017;16:393.

30. Bidlingmayer $\mathrm{WL}$. The measurement of adult mosquito population changes-some considerations. J Am Mosq Control Assoc. 1985:1:328-48.

31. Bidlingmayer WL, Evans DG, Hansen CH. Preliminary study of the effects of wind velocities and wind shadows upon suction trap catches of mosquitoes (Diptera: Culicidae). J Med Entomol. 1985;22:295-302.

32. Charlwood JD, Paru R, Dagoro H. Raised platforms reduce mosquito bites. Trans R Soc Trop Med Hyg. 1984;78:141-2.

33. Muller GC, Beier JC, Traore SF, Toure MB, Traore MM, Bah S, et al. Successful field trial of attractive toxic sugar bait (ATSB) plant-spraying methods against malaria vectors in the Anopheles gambiae complex in Mali, West Africa. Malar J. 2010;9:210

34. Zhu L, Müller GC, Marshall JM, Arheart KL, Qualls WA, Hlaing WM, et al. Is outdoor vector control needed for malaria elimination? An individualbased modelling study. Malar J. 2017;16:266.

\section{Publisher's Note}

Springer Nature remains neutral with regard to jurisdictional claims in published maps and institutional affiliations.
Ready to submit your research? Choose BMC and benefit from:

- fast, convenient online submission

- thorough peer review by experienced researchers in your field

- rapid publication on acceptance

- support for research data, including large and complex data types

- gold Open Access which fosters wider collaboration and increased citations

- maximum visibility for your research: over 100M website views per year

At BMC, research is always in progress.

Learn more biomedcentral.com/submissions 